\title{
Práticas transversais do Apoiador Institucional em territórios de produção de saúde
}

\author{
Cross-sectional practices of the Institutional Supporter in territories \\ of health production
}

\section{Prácticas transversales del Apoyo Institucional en territorios de producción de salud}

Camila Cristina de Oliveira Rodrigues ${ }^{1}$

Sergio Resende Carvalho ${ }^{2}$

\begin{abstract}
RESUMO: O trabalho de Apoio tem cumprido um papel relevante, que pode ser assumido de distintas formas e em diferentes coletivos. Pretende-se, neste ensaio, apresentar a experiência do Apoiador Institucional e seu fazer na rede de saúde da cidade de Campinas, São Paulo, Brasil. Serão discutidas, mais especificamente, a prática de apoio que vem sendo desenvolvida junto à Rede de Atenção Psicossocial e algumas estratégias utilizadas pelos profissionais para desempenhar esse trabalho pioneiro. Nesse sentido, destacam-se ações como a discussão de casos, a realização de atendimentos compartilhados e o acompanhamento de visitas domiciliares como ferramentas fundamentais do trabalho desempenhado pelo Apoiador Institucional. Busca-se compartilhar a prática que atravessa essa experiência por meio da apresentação de um relato de caso articulado pelo fazer do apoiador. Com base nas concepções dos pensadores da diferença, essa experiência foi cartografada aos moldes de uma narrativa e nos permitiu constatar o caráter transversal do trabalho do apoiador e a potencialidade de produção de redes presente nessa prática.
\end{abstract}

Palavras-Chave: Gestão em Saúde, Apoio ao Planejamento em Saúde, Saúde Pública, Saúde Mental, Relatos de Casos.

\begin{abstract}
The work of Support has fulfilled a relevant role that, which can be assumed in different ways and in different collectives. It is intended, in this essay, to present the experience of the Institutional Supporter and its work in the health network of the city of Campinas, São Paulo, Brazil. It will be discussed, more specifically, the practice of support that has been developed with the Network of Psychosocial Care and some strategies used by professionals to carry out this pioneering work. In this sense, actions such as the discussion of cases, the accomplishment of shared care and the follow-up of home visits as fundamental tools of the work carried out by the Institutional Supporter are highlighted. It seeks to share the practice that goes through this experience through

1 Psicóloga formada pela Universidade Estadual Paulista (UNESP - Campus de Assis/SP), Especialista em Saúde Mental pela Universidade Estadual de Campinas (UNICAMP), Gestora do CAPS ad III Independência do município de Campinas/SP e Mestra em Saúde Coletiva pela Faculdade de Ciências Médicas (UNICAMP). Universidade Estadual de Campinas (UNICAMP). Campinas, São Paulo, Brasil. E-mail: camila.rodrigues888@gmail.com

2 Médico formado pela Universidade de Minas Gerais (UFMG). Docente do Departamento de Saúde Coletiva da Faculdade de Ciências Médicas FCM - UNICAMP. Coordenador da Linha de Pesquisa Política da Subjetividade. Universidade Estadual de Campinas (UNICAMP). Campinas, São Paulo, Brasil. E-mail: 2srcarvalho@gmail.com
\end{abstract}

ISSN 1982-8829 Tempus, actas de saúde colet, Brasília, 11(3), 232-246, 2017 - Epub mar, 2018 
the presentation of a case report articulated by the work of the supporter. Based on the conceptions of the thinkers of difference, this experience was mapped to the model of a narrative and allowed us to verify the transversal character of the work of the supporter and the potential of network production present in this practice.

KeyWords: Health Management, Health Planning Support, Public Health, Mental Health, Case Reports.

RESUMEN: El trabajo de Apoyo ha cumplido un papel relevante que puede ser asumido de distintas formas y en diferentes colectivos. En este ensayo, pretendemos presentar la experiencia del Apoyente Institucional y su hacer en la red de salud de la ciudad de Campinas, São Paulo, Brasil. Discutiremos más específicamente la práctica de apoyo que viene siendo desarrollada junto a la Red de Atención Psicosocial y algunas estrategias utilizadas por los profesionales para desempeñar ese trabajo pionero. En este sentido, destacamos acciones como la discusión de casos, la realización de atendimientos compartidos y el acompañamiento de visitas domiciliarias como herramientas fundamentales del trabajo desempeñado por el Apoyente Institucional. Buscamos compartir la práctica que atraviesa esa experiencia por medio de la presentación de un relato de caso articulado por el hacer del sostenedor. Con base en las concepciones de los pensadores de la diferencia, esa experiencia fue cartografiada a los moldes de una narrativa y nos permitió constatar el carácter transversal del trabajo del apoyador y la potencialidad de producción de redes presente en esa práctica.

Palabras clave: Gestión en Salud, Apoyo a la Planificación en Salud, Salud Pública, Salud Mental, Relatos de Casos. 
“À diferença da informação, o relato não se preocupa em transmitir o puro em si do acontecimento, ele o incorpora na própria vida daquele que conta, para comunicá-lo como sua própria experiência àquele que escuta. Dessa maneira o narrador nele deixa seu traço, como a mão do artesão no vaso de argila."

Walter Benjamin

ISSN 1982-8829 Tempus, actas de saúde colet, Brasília, 11(3), 232-246, 2017 - Epub mar, 2018 


\section{INTRODUÇÃO}

\section{A Estratégia de Apoio nas Redes de Saúde}

A implantação da Estratégia de Apoio na Saúde Pública vem ao encontro de outros esforços de construção de um processo de trabalho participativo e compartilhado no SUS, tais como as Reuniões de Equipe, os Conselhos de Saúde e os Colegiados Gestores. Dessa maneira, entende-se que o Apoio possibilita a construção de um campo de problematização sobre as tradicionais formas de relações existentes no mundo do trabalho e as verdades pré-concebidas sobre os modos de adoecimento e tratamento ofertados aos sujeitos ${ }^{1}$.

Por se tratar de uma proposta inovadora, as diretrizes conceituais e de aplicação dessa estratégia vêm sendo desenvolvidas por muitos autores, como Pena ${ }^{2}$, Oliveira ${ }^{3}$, Dorsa ${ }^{4}$ e, sobretudo, por Campos $^{5}$, que desdobrou essa proposta a partir da construção do Método Paidéia e da proposta de Cogestão de Coletivos ${ }^{6}$.

Nesse sentido, o Apoio visa ao fortalecimento de uma prática que coletiviza e amplia as ações de saúde, tornando-se um diferencial na implantação das redes de cuidado. Assim, de modo complementar às demais frentes que compõem as práticas em saúde, espera-se que o apoiador acompanhe ativamente as ações estratégicas desempenhadas pelas equipes. Acredita-se que os espaços privilegiados de intervenção dos apoiadores são os territórios onde trabalhadores e usuários estão inseridos, já que esses são os locais onde é possível reconhecer e intervir "in ato" sobre os modelos de assistência prestada aos usuários e sobre o processo de trabalho desempenhado pelas equipes de saúde ${ }^{2}$.

Segundo Oliveira, "o objeto de trabalho do apoiador é o processo de trabalho de coletivos que se organizam para produzir saúde. (...) Opera em 'uma região limítrofe entre a clínica e a política, entre o cuidado e a gestão - lá onde estes domínios se interferem mutuamente"3.

Assim, é fundamental no trabalho de Apoio reconhecer que as ações de cuidado estão atravessadas, também, por um campo de virtualidades, onde se podem reconhecer processos de construção imaginária do ato de cuidar e das formas de cuidado. Um exemplo poderia ser aquele usuário que insiste pela realização de uma radiografia, como se esse fosse o ato determinante, ou marcador, de que está sendo cuidado. $\mathrm{O}$ apoiador, nesse caso, precisa estar advertido de que existe uma construção imaginária de demandas. Trata-se de uma operação onde os registros imaginários atravessam o plano virtual para o plano real, atualizando no campo do simbólico que é um veículo de expressão privilegiado nas relações humanas ${ }^{7}$.

Segundo FRANCO E MERHY, o usuário, “ao demandar o procedimento está acessando em nível imaginário, aquele universo simbólico que dá significado amplo ao procedimento, atribuindolhe uma potencialidade que ele não tem, que é a de produzir o cuidado por si mesmo" " Isso opera 
da mesma maneira para os trabalhadores e seus processos de trabalho, pois muitas vezes eles se reconhecem apenas como encaminhadores ou ofertadores de procedimentos, que, muitas vezes, são pouco resolutivos e calcados apenas no modelo tecnoassistencial médico.

Dessa forma, "vamos percebendo, portanto, que a produção imaginária da demanda é uma construção sócio-histórica que tem sua gênese no modo como foram constituídos os modelos tecnoassistenciais para a saúde" ". Portanto, as demandas que são endereçadas ao apoiador devem ser entendidas como os elementos analíticos pelos quais ele também poderá acessar a dinâmica dos usuários e das equipes de saúde.

Entende-se que é a partir do acolhimento e da análise das demandas que ele poderá construir os meios de intervenção, novas pactuações e contratualidades que podem ser articuladas com objetivo de compor um meio institucional que suscite a capacidade de invenção de outras tecnologias de trabalho e produção de cuidado.

Neste sentido, reconhece-se que a prática do Apoiador possui uma perspectiva multicêntrica, pois sua atuação tem alcance tanto na dimensão que trata mais diretamente da produção de tecnologias de cuidado que atendam melhor às necessidades dos usuários; quanto na dimensão de ampliação da capacidade analítica e operacional das equipes e das redes de saúde, uma vez que provoca a possibilidade de elas operarem de modo mais criativo, inventivo e fluido ${ }^{4}$.

\section{DESENVOLVIMENTO}

\section{A estratégia de apoio aplicada no município de Campinas/SP}

No município de Campinas, a Rede de Saúde (SUS) vivencia uma experiência da Estratégia do Apoio tanto no nível distrital (denominada Apoio Institucional) como no nível local (denominada Apoio Matricial). A composição dessas modelagens da Estratégia de Apoio vem sendo debatida tanto pela rede de saúde quanto pelas universidades, onde muitas pesquisas vêm sendo desenvolvidas com intuito de aprofundar o conhecimento teórico sobre tais práticas, assim como de avaliar os alcances de sua operacionalização na rede SUS Campinas ${ }^{2-3-4-8}$.

O Apoio Matricial é uma estratégia que teve grande adesão da Rede de Atenção Psicossocial de Campinas, em detrimento de outras especialidades da área da saúde que possuem experiências ainda bastante tímidas. No campo da Saúde Mental, essa prática ficou popularmente conhecida como "Matriciamento" e vem sendo desempenhada pelos profissionais dos diferentes serviços da rede.

Os encontros de "Matriciamento", em geral, acontecem por meio de reuniões de discussão de casos; debates sobre temáticas de saúde mental e atendimentos compartilhados, que podem ocorrer na unidade ou através de visitas domiciliares realizadas em conjunto. Grande parte das 
equipes aponta o "Matriciamento" como uma ação significativa para os serviços, considerando que houve maior aproximação entre as realidades das diferentes unidades, bem como uma compreensão mais ampliada sobre a necessidade de romper com a fragmentação do cuidado e criar estratégias de trabalho compartilhado, visando à atenção integral dos usuários ${ }^{4}$.

Com relação ao Apoio Institucional, este se dá mediante a constituição de uma equipe multiprofissional situada em um nível de gestão. A equipe de Apoiadores Institucionais costuma ser composta por profissionais com grande experiência, tanto em suas áreas de concentração quanto na política pública de saúde, e é conduzida por um diretor, que fica responsável pelo acompanhamento do trabalho do Distrito Sanitário.

O município de Campinas está dividido em cinco Distritos Sanitários coordenados pela gestão central da Secretaria Municipal de Saúde. Cada Distrito é responsável por um conjunto de bairros, com características epidemiológicas específicas, que marcam com suas especificidades a singularidade de cada território distrital. As características epidemiológicas de cada Distrito costumam ser utilizadas como instrumento na construção de diagnósticos de intervenção sobre cada território.

Neste sentido, está a cargo de cada Distrito Sanitário: planejar tanto a implantação como a operacionalização dos equipamentos de saúde do seu território de abrangência; acompanhar o trabalho desenvolvido nos territórios através de ações conjuntas e da produção de indicadores de saúde; e articular junto à Secretaria Municipal de Saúde os recursos humanos e materiais necessários para efetivação e qualificação dos serviços prestados à população.

No que diz respeito ao trabalho do Apoiador Institucional no campo da Saúde Mental, cabe ao mesmo acompanhar os Colegiados de Gestão da área entre outros Fóruns Municipais que discutam temáticas específicas da Saúde Mental, contribuindo tanto com o desenvolvimento das políticas públicas municipais quanto com a disseminação dos debates para os serviços do seu território distrital, suscitando com eles discussões críticas que aumentem sua capacidade de análise e protagonismo nas ações locais, interinstitucionais e intersetoriais.

Além disso, é importante que esse profissional se faça presente no cotidiano dos equipamentos do seu território distrital, promovendo encontros tanto com as equipes da Atenção Básica quanto com os serviços especializados da Saúde Mental, como os CAPS III, CAPS Ad, CAPS-i, Serviços Residenciais Terapêuticos, Oficinas de Geração de Renda, Centros de Convivência, entre outros. O trânsito do Apoiador nessa rede é de fundamental importância para a formação de vínculo com as equipes, bem como para a elaboração de diagnósticos institucionais e produção de intervenções qualificadas ${ }^{2}$.

Contudo, sustentar uma presença ativa nos serviços não é uma tarefa fácil e requer ISSN 1982-8829 Tempus, actas de saúde colet, Brasília, 11(3), 232-246, 2017 - Epub mar, 2018 
grande capacidade de planejamento, pois as agendas dos Apoiadores são preenchidas, em geral, com demandas diversificadas vindas, muitas vezes, de última hora, tanto por parte da Secretaria Municipal de Saúde quanto das unidades que estão sob sua referência direta. Assim, os cronogramas de atividades dos Apoiadores Institucionais constituem verdadeiros mapas cartográficos que podem, inclusive, expressar dados interessantes sobre o contexto atual dos serviços de saúde do seu território.

Desse modo, o trabalho do Apoiador Institucional em Campinas acontece por meio de um tipo de prática que se dá em uma espécie de fronteira existente entre a clínica e a gestão. Atuando junto às equipes, principalmente a partir de demandas de articulação de casos muito complexos ou de situações embaraçosas, o apoiador é convidado a experienciar constantes desafios com as equipes. Cada nova demanda que se apresenta ao Apoiador traz consigo uma novidade que atualiza a necessidade de invenção contínua da prática em saúde, e quanto mais o profissional nesse papel estiver aberto e disposto a esse campo de criação, maior será a sua capacidade de composição com as equipes que ele acompanha ${ }^{3}$.

É comum que esse trabalho seja disparado por meio de um pedido de reunião de discussão de caso. No entanto, é importante ressaltar que as equipes esperam que o apoiador não se coloque em um papel de supervisão do trabalho desempenhado por elas, mas, sim, como um colaborador que protagoniza ações compartilhadas, articulações com outros dispositivos desconhecidos ou ignorados, arejando os espaços desgastados pela repetição das práticas e esgarçados pela tensão do manejo de situações complexas ${ }^{1}$.

Nesse sentido, concebe-se que a prática exercida pelo Apoiador Institucional pode qualificar a gestão do cuidado a partir da construção de uma rede de atenção que o coletivo de trabalho, articulado pela ação de apoio, agencia e compõe. Nessa experiência, interroga-se a lógica de encaminhamento, uma vez que se realiza uma oferta de coprodução de cuidado para as equipes de saúde, além de um suporte continuado desse profissional externo à equipe, que reforça a rede de cuidados articulada para e com os usuários.

É importante ressaltar que, apesar de, neste trabalho, estar-se discutindo a prática do apoiador institucional no campo da saúde mental, entende-se que o apoio não é um papel restrito ao Apoiador Institucional, mas, sim, uma função estratégica que pode ser assumida por outros trabalhadores de saúde. Portanto, esse fazer-apoio pode ter diferentes semblantes institucionais, sobretudo, se for tomado enquanto um acontecimento que atravessa os encontros de produção de saúde 5 .

Através da narrativa de um caso analisador ${ }^{3}$, procurar-se-á elucidar algumas das estratégias

3 O caso apresentado é um caso fictício construído com base nas histórias reunidas pela experiência vivida no acompanhamento de muitos casos em que atuaram os autores deste artigo enquanto trabalhadores e apoiadores da rede de saúde mental do município de Campinas-SP.

ISSN 1982-8829 Tempus, actas de saúde colet, Brasília, 11(3), 232-246, 2017 - Epub mar, 2018 
que podem ser utilizadas pelos Apoiadores Institucionais no cotidiano do seu trabalho. Apostouse que por meio de uma narrativa se poderia mapear de modo mais intensivo os movimentos empreendidos por esses profissionais junto aos trabalhadores e usuários dos serviços territoriais, além de cartografar suas práticas de modo a compartilhar esse fazer com outros profissionais e pesquisadores.

Acredita-se que essa maneira de discutir o trabalho do Apoiador Institucional é um método privilegiado de construir pesquisa em saúde, uma vez que ele captura um retrato da rede acontecendo. A narrativa de caso, portanto, apresenta os encontros das equipes com as necessidades reais da população do território, constituindo um regime de luz que evidencia as dificuldades, fragilidades e complexidades que tocam os processos de promoção de saúde.

\section{O caso Ana como dispositivo analisador da prática do apoio enquanto uma estratégia marginal de produção de saúde ${ }^{1}$}

Dona Ana tinha cerca de 60 anos de idade e havia perdido o marido há pouco tempo. Fora abandonada pela filha mais velha, que teria sido encarregada dos cuidados da família a pedido do pai. Ana tinha um histórico de internações psiquiátricas anteriores e vivia, naquele momento, com um dos filhos, que tinha por volta de 30 anos e apresentava um diagnóstico de Retardo Mental e uso abusivo de álcool.

A usuária apresentava, desde a morte do marido, um quadro clínico mais descompensado (Diabetes e Hipertensão) e tinha dificuldades de se alimentar adequadamente, uma vez que a filha saíra de casa levando consigo parte significativa do recurso financeiro da família, proveniente do benefício deixado pelo pai.

A Equipe de Saúde da Família responsável pelo cuidado de Dona Ana já havia tentado uma série de estratégias, a fim de acompanhar a saúde da usuária e de promover melhores condições de vida. Visitas domiciliares para orientação do acompanhamento da Diabetes e da Hipertensão eram frequentemente realizadas; articulações com vizinhos para auxílio na administração da medicação e da alimentação também foram tentadas, mas a comunidade também reportava para a equipe as suas limitações.

Dona Ana permanecia apresentando dificuldade no tratamento. Articulações com outros serviços também foram tentadas, porém, houve poucos avanços no sentido de prover algum auxílio com relação às necessidades de cuidado diário. Os problemas familiares de Dona Ana trouxeram maiores dificuldades para sua saúde, preocupando parte dos vizinhos e da equipe de saúde frente aos riscos de morte a que a usuária vinha sendo submetida.

Assim, o processo de agravamento de suas condições de saúde constituiu um campo de tensão entre vizinhos e equipe, que, diante da dificuldade de encontrar outras estratégias resolutivas, 
optou por solicitar ao Ministério Público o abrigamento da usuária em uma instituição para idosos que garantisse seu acompanhamento clínico diário.

Considerando a condição em que Dona Ana se encontrava e levando em consideração que era egressa de uma internação em Hospital Psiquiátrico, a determinação expedida pela instância judicial, em consonância com parte da gestão da Secretaria de Saúde, definiu que a paciente deveria ser encaminhada imediatamente para nova internação em instituição de saúde mental. É nesse momento que o Apoiador foi acionado na estratégia de articulação de cuidado do caso pela equipe de Apoiadores Institucionais do Distrito Sanitário responsável pela região onde Dona Ana morava. A solicitação feita ao Apoiador era de que acompanhasse a equipe do Centro de Atenção Psicossocial (CAPS) de referência na realização do encaminhamento de Dona Ana até o hospital onde ela permaneceria internada.

Contudo, a construção de um encaminhamento adequado para essa demanda só pôde ser delineada pelo Apoiador após a realização de algumas visitas domiciliares feitas à usuária em conjunto com as equipes da Unidade Básica de Saúde (UBS) e do CAPS. Através das visitas domiciliares, pareceu ao apoiador que Dona Ana necessitava de um acompanhamento mais intensivo que atendesse às suas necessidades frente ao agravamento de saúde, tanto do ponto de vista clínico como mental, porém, ele acreditava que esse trabalho poderia ser realizado em serviços territorializados, não havendo justificava técnica para que isso tivesse que ser realizado por meio de uma internação psiquiátrica.

Acredita-se que a implicação ativa do Apoiador Institucional com o caso em questão foi determinante para que ele produzisse um posicionamento próprio e qualificado sobre as reais necessidades da usuária em questão. A orientação técnica construída pelo apoiador por meio das ações que coproduziu com as equipes de saúde precisou ser fortemente sustentada durante as reuniões que definiram a construção do projeto terapêutico do caso. Constatou-se, no decorrer dos encontros, a presença de discursos divergentes entre as equipes envolvidas, assim como demandas de intervenção diversificadas que tendiam desde a institucionalização definitiva e imediata da usuária até o não reconhecimento da necessidade de uma medida de saúde para o caso, argumento que era justificado com base na problemática social proeminentemente manifestada pelo caso em questão.

As articulações que viabilizaram a condução desse caso por parte do Apoiador Institucional envolveram desde encontros com o Departamento Jurídico da Secretaria Municipal de Saúde e do Ministério Público até reuniões com as equipes da Unidade Básica, o CAPS e técnicos da Assistência Social de diversos níveis de complexidade. Em articulação com o CAPS, o Apoiador construiu a inserção de Dona Ana em uma vaga de leito-noite, em resposta ao pedido de internação psiquiátrica da usuária. Dona Ana foi, então, inserida no CAPS, onde foi acolhida e avaliada, passando a participar de oficinas terapêuticas e a receber cuidado de uma equipe multiprofissional. 
Suas idas ao serviço foram garantidas através da articulação com a rede de Assistência Social, que disponibilizou um transporte para levá-la. Com relação aos cuidados clínicos (hipertensão e diabetes), a Unidade Básica de Saúde deu continuidade ao acompanhamento da usuária por meio de consultas e visitas domiciliares. Assim, nos dias em que a usuária ia ao CAPS, suas medicações (clínicas e psiquiátricas) eram feitas pela equipe do CAPS, e nos dias em que Dona Ana estava em sua casa, os vizinhos e a Unidade Básica de Saúde lhe davam esse apoio.

Em construção com as equipes dos diversos serviços e a gestão de diversos níveis das Secretarias de Saúde e Assistência, bem como com o Ministério Público, trabalhou-se sob a tensão de responder ao instituído sem perder a possibilidade de construção de um campo instituinte que problematizasse as orientações determinadas para a condução desse caso, em especial, as advindas dos discursos médico e jurídico.

Assim, em se tratando de um momento histórico, em que as Internações Compulsórias reaparecem enquanto alternativa para solucionar situações complexas de vulnerabilidades sociais diversas, o trabalho do Apoiador Institucional pode imprimir na ação dos diversos atores uma mediação que produziu outro entendimento e direcionamento de cuidado ${ }^{9}$. Esse trabalho possibilitou, ainda, que um processo de coprodução de uma intervenção clínica, psicossocial e assistencial pudesse ser realizado, garantindo as premissas de um cuidado em saúde mental territorializado e em liberdade.

Além disso, através do caso de Dona Ana, a relação entre as equipes da Atenção Básica, da Atenção Especializada em Saúde Mental (CAPS) e da rede de Assistência Social territorial foi progressivamente ressignificada. Os sucessivos ensaios de aproximação e contato entre os serviços foram mediados pela figura do apoiador, que colaborou para que as impressões distorcidas e as fantasias constituídas entre as equipes fossem dissolvidas de modo que os serviços pudessem se reposicionar sobre o trabalho desempenhado nos casos compartilhados.

A necessidade de articulação intersetorial também emergiu no campo dos tensionamentos das equipes com relação ao trabalho do apoiador, especialmente no que se refere à capacidade atual das equipes de outros setores públicos de se ocuparem das necessidades da população. Desse modo, foi no jogo de forças entre os limites e as possibilidades de ação de cada instituição envolvida com o caso de Dona Ana que o Apoiador pôde explorar a rede existente, seus recursos e falhas, seus pontos potentes e seus nós enrijecedores de produção de linhas de cuidado.

Passos, Kastrup e Escóssia ${ }^{10}$ afirmam que o que está em jogo na produção de saúde é a construção de uma política que atue em favor da vida como potência, e não aprisionamento das formas desejantes ou dos processos de subjetivação. Assim, a produção de desvios enquanto um traço característico do contemporâneo deveria orientar as práticas de saúde, desviando os modos 
de cuidar de práticas que subjuguem a singularidade da existência a determinados modelos e normações históricas.

Deste modo, aposta-se na estratégia do apoio como uma prática capaz de ser desenvolvida na transversalidade das ações em saúde e de se orientar por uma diretriz ético-clínico-política que afirma a diferença e os movimentos desviantes realizados pelos usuários, investindo em que as relações de produção de cuidado possam engendrar processos de ressingularização da existência.

\section{DISCUSSÃO}

\section{A potencialidade da estratégia de apoio}

A partir da experiência relatada, entende-se que a prática do apoio tem um lugar estratégico na composição de uma rede de saúde, que pode ser potencializado especialmente se lograr contribuir para a construção de uma prática transversal que agencia outras redes e conexões de saúde possíveis na intercessão das políticas, das práticas e dos equipamentos do SUS.

Neste sentido, o apoio se alinha às concepções de Guattari ${ }^{11}$ quando afirma ser:

(...) essencial que se organizem (...) novas práticas micropolíticas e microssociais, novas solidariedades, uma nova suavidade juntamente com novas práticas estéticas e novas práticas analíticas das formações do inconsciente. Parece-me que essa é a única via possível para que as práticas sociais e políticas saiam dessa situação, quero dizer, para que elas trabalhem para a humanidade e não mais para um simples reequilíbrio permanente do Universo das semióticas capitalísticas ${ }^{11}$.

Dessa maneira, ao se produzir, enquanto uma prática inovadora e transversal, o Apoio pode romper com as concepções mais duais que ainda dividem o campo da saúde, buscando um movimento de ressingularização do seu processo de trabalho, valorizando a produção criativa de ações que afirmem a potência de vida dos sujeitos que buscam cuidado no SUS ${ }^{12}$.

Pena $^{2}$, a partir de suas investigações junto ao campo da Saúde Mental, considera que:

(...) afirmar a inseparabilidade entre gestão e clínica é afirmar a gestão (...) enquanto uma prática de cuidado; uma prática que busca dar suporte ao processo de invenção do cotidiano que é operado pela clínica. Para isso, devemos compreender a gestão como desdobramento do cotidiano, uma função que cuida do trabalho em saúde mental como um modo de criar espaços comuns ou zonas de vizinhança entre trabalhadores e usuários dos serviços ${ }^{2}$.

O Apoio, portanto, propõe ações junto às equipes de saúde visando à articulação de uma clínica mais ampliada que aposta que ações de gestão são exercidas por todos os sujeitos que atuam na rede de saúde, inclusive os próprios usuários, que devem ser tomados, também, como cogestores dos seus projetos terapêuticos ${ }^{6}$. 
Assim, concebe-se o trabalho do Apoiador como uma função do campo da micropolítica do trabalho em saúde que pode engendrar movimentos e processos de diferenciação nos modos de se posicionar e agir das equipes e de outros setores envolvidos nas políticas públicas ${ }^{13}$.

Através de um fazer implicado com a demanda das instituições, o Apoiador abre brechas para outros regimes de sensibilidade e dizibilidade nos sujeitos que compõem as instituições, possibilitando que o processo de trabalho seja construído e analisado coletivamente, de modo a ressignificar o papel e o comprometimento das equipes com a produção da integralidade do cuidado.

Muitas vezes, para as instituições e para a rede, o Apoiador será o profissional chamado à tarefa de "indicar algo de fora", sendo, portanto, um elemento institucional que carrega em si uma função paradoxal de pertencimento e não pertencimento.

Contudo, ao transitar nas fronteiras institucionais e investir sua observação e análise na virtualidade presente nos processos de ação e relação entre os diferentes sujeitos, não é com "algo de fora" que o Apoiador contribuirá para o trabalho das equipes. Trata-se, sim, de "outro posicionamento" e "outra perspectiva" sobre a problemática vivenciada pelas equipes de saúde, que não é da ordem do plano da individualização, mas, sim, da singularização que se expressa em cada demanda que chega ao apoiador.

Operar mudanças em conjunto com as equipes implica ao apoiador também criar dispositivos que provoquem os sujeitos a construir outras reflexões sobre a problemática vivenciada e a desejar ativamente algumas mudanças. Como dispositivos, pode-se entender desde uma análise de dados epidemiológicos até a criação ou a reformulação desses espaços institucionais tradicionais, desde que o que se crie leve à ampliação dos gradientes de diálogo e trocas no serviço, favorecendo a construção coletiva e de produção comum naquele território de ação em saúde.

É importante destacar que se trata de um trabalho processual, que requer tempo e investimento na construção de vínculo e confiança com as equipes, de modo que se crie um campo de abertura onde os movimentos de resistência, as interdições, os temas, as ações e relações veladas possam emergir e ser trabalhados ${ }^{5-14}$.

O campo de atuação do Apoiador, portanto, é composto por uma bricolagem de aspectos que operam significações e subjetivações nas pessoas e nas instituições envolvidas com problemáticas sociais complexas, que, na maioria das vezes, tensionam a capacidade de resolubilidade das ações de saúde vigentes ${ }^{14}$.

À luz do caso apresentado, procura-se cartografar alguns dos possíveis movimentos empreendidos a partir do trabalho de um Apoiador Institucional. Trata-se de um trabalhador que, muitas vezes, experimenta um papel de se colocar e/ou ser colocado em um "não lugar", pois ele 
não é um membro da equipe com quem ele está atuando e também não é um membro de setores mais centrais que poderiam dar respostas mais efetivas de intervenção para as ações sobre as quais o apoiador é convidado a atuar. Por outro lado, o trabalho junto às equipes, por meio de uma prática de compartilhamento de casos, possibilita a construção de outro papel, que diz respeito "àquele profissional que pode estar ao lado das equipes" coproduzindo uma narrativa híbrida que, muitas vezes, implica uma desconstrução sobre os modos e as expectativas que diversos atores produzem sobre as demandas apresentadas.

Neste sentido, a ação do Apoio nos parece alinhada à proposta de Passos, Kastrup e Escóssia ${ }^{10}$ quando se indica que tecer uma narratividade sobre um caso deve implicar uma operação de desmontagem do caso. A montagem de um caso conduz a uma representação da realidade desde um lugar externo, enquanto a desmontagem opera como um analisador dos movimentos da rede que permite desconstruir verdades naturalizadas. Essa modalidade de ação nos parece de grande importância para produção de rede de saúde, na medida em que se realiza na intercessão do fazer coletivo das equipes e contribui para a construção de relações de trabalho mais compartilhadas e coletivizadas. A intermediação exercida pelo Apoiador, dessa forma, modifica o campo das relações de força e saber instituídas nas equipes de saúde e nos múltiplos arranjos institucionais.

O Apoiador, portanto, é concebido enquanto um trabalhador cuja prática pode ser um dispositivo capaz de atravessar estruturas mais sedimentadas dos modos de produção de saúde para criar brechas e estratégias instituintes que favoreçam aberturas de um meio que estimule a arte de inventar formas de cuidado singularizado frente às situações complexas.

Com isso, acredita-se que o Apoio pode contribuir muito para a construção das redes de saúde, favorecendo o movimento de fortalecimento do SUS e o desenvolvimento de modelos de gestão mais coletivizados e compartilhados. Por essas razões, entende-se que a prática do apoio e a constituição de grupos de apoiadores devem ser mais estimuladas pelas políticas e redes de saúde de todo país 6 .

\section{CONSIDERAÇÕES FINAIS}

Se a produção de saúde, em muitos momentos, foi pensada através de linhas dicotômicas que a dividiram entre as políticas públicas e as práticas de saúde, a gestão e a assistência, o trabalhador de saúde e o usuário, no cotidiano de cada serviço, defronta-se com acontecimentos engendrados nos encontros entre pessoas e tecnologias que se delineiam em um composto singular chamado de cuidado. O cuidado enquanto acontecimento é atravessado incessantemente por dimensões que vão desde as necessidades sociais, a constituição e os modos de circulação e subjetivação dos sujeitos nas cidades, os conhecimentos e procedimentos científicos aplicados à saúde, até as relações de poder e governamentalidade ${ }^{15-164}$.

$4^{2}$ Entende-se por governamentalidade as táticas de governo que permitem definir o que deve ou não competir ao Estado $^{15}$.

ISSN 1982-8829 Tempus, actas de saúde colet, Brasília, 11(3), 232-246, 2017 - Epub mar, 2018 
Desta forma, as políticas públicas e as práticas de saúde podem ser tomadas enquanto dispositivos abertos e transversais que se encontram em permanente reestruturação. Trata-se de um campo de forças composto por uma heterogeneidade de elementos que experimentam no encontro tenso entre o instituído e o instituinte a possibilidade de produzir expressões do que a contemporaneidade pode reconhecer como tecnologias de cuidado $^{12}$.

Neste sentido, discutir a produção de saúde sob a perspectiva da transversalidade vem de encontro com o projeto de sustentação do SUS, que, enquanto modelo universal de saúde, visa à construção de políticas públicas e práticas de cuidado alinhadas à luta por um processo de democratização no Brasil, que aponta respostas às crises sociais vigentes por meio da cidadania e dos direitos sociais ${ }^{2-3-8}$.

Assim, entende-se que a experiência do trabalho que vem sendo desenvolvido no SUS através do Apoio Institucional é consonante com este debate, pois a Estratégia de Apoio, enquanto uma prática que se dá na transversalidade das relações estabelecidas entre a gestão, a equipe e os usuários dos serviços, é uma prática que rompe com as concepções mais duais sobre o campo da saúde, buscando um movimento de ressingularização para os processos de trabalho do SUS ${ }^{6}$.

\section{REFERÊNCIAS:}

1. Campos GWS. Equipes de referência e apoio especializado matricial: um ensaio sobre a reorganização do trabalho em saúde. Ciência e Saúde Coletiva. 1999; 4 (2): 393-403.

2. Pena RS. O Apoio institucional como estratégia de gestão em coletivos na saúde mental [tese]. Campinas: Universidade Estadual de Campinas, Faculdade de Ciências Médicas; 2012. p. 192.

3. Oliveira GN. Devir apoiador: uma cartografia da função apoio. [tese]. Campinas: Universidade Estadual de Campinas, Faculdade de Ciências Médicas; 2011. p.34.

4. Dorsa M. Saúde mental na atenção básica: um estudo hermenêutico-narrativo sobre o apoio matricial no SUS Campinas [Dissertação]. Campinas: Universidade Estadual de Campinas, Faculdade de Ciências Médicas; 2006.

5. Campos GWS. Paidéia e gestão: um ensaio sobre o Apoio Paidéia no trabalho em saúde. 2003. Available from: $<$ www.gastaowagner.com.br $>$

6. Campos GWS. Um método para análise e co-gestão de coletivos. São Paulo: Hucitec; 2013.

7. Franco TB, Merhy, EE. Produção Imaginária da Demanda. In: Pinheiro R, Mattos RA. (orgs.). Construção Social da Demanda. Rio de Janeiro: IMS/UERJ-CEPESC-ABRASCO; 2005. p. 5.

8. Souza TP. A norma da abstinência e o dispositivo drogas: direitos universais em dispositivos marginais de produção de saúde [tese]. Campinas: Universidade Estadual de Campinas, Faculdade de Ciências Médicas; 2013. 
9. Rodrigues, CCO. Uma genealogia dos anormais na contemporaneidade: ensaios sobre alguns processos de judicialização que atravessam a Rede de Atenção Psicossocial [dissertação]. Campinas: Universidade Estadual de Campinas, Faculdade de Ciências Médicas; 2017.

10. Passos E, Kastrup V, Escóssia L (Orgs.). Pistas do método da cartografia: pesquisa-intervenção e produção de subjetividade. Porto Alegre: Editora Sulina; 2009.

11. Guatarri F. As três ecologias. Campinas: Papirus; 2001. Available from: $<$ http://claudioulpiano.org.br.s87743. gridserver.com/wpcontent/uploads/2012/04/guattari-as-tres-ecologias3.pdf> p. 35.

12. Guatarri F. Psicanálise e Transversalidade: ensaios de análise institucional. São Paulo: Ideias \& Letras; 2004.

13. Guatarri F, Rolnik S. Micropolítica: cartografias do desejo. Rio de Janeiro: Vozes; 1993.

14. Merhy EE. O ato de governar as tensões constitutivas do agir em saúde como o desafio permanente de algumas estratégias gerenciais. Available from: $<$ http://www.uff.br/saudecoletiva/professores/merhy/indexados-10.pdf $>$

15. Foucault M. Microfísica do poder. Rio de Janeiro: Graal; 1986. A governamentalidade; p. 163-72.

16. Merhy EE. O cuidado é um acontecimento e não um ato. Available from: $<\underline{\text { http://www.uff.br/saudecoletiva/ }}$ professores/merhy/capitulos-17.pdf>

Relato apresentado em 29/10/2017

Relato aprovado em 09/01/2018

Relato publicado no sistema em 16/04/2018

ISSN 1982-8829 Tempus, actas de saúde colet, Brasília, 11(3), 232-246, 2017 - Epub mar, 2018 